\title{
Automatic detection and mapping of solar photovoltaic arrays with deep convolutional neural networks in high resolution satellite images
}

DOI:

10.1109/EI250167.2020.9347211

\section{Document Version \\ Accepted author manuscript}

Link to publication record in Manchester Research Explorer

Citation for published version (APA):

$\mathrm{He}, \mathrm{K}$., \& Zhang, L. (2021). Automatic detection and mapping of solar photovoltaic arrays with deep convolutional neural networks in high resolution satellite images. In 2020 IEEE 4th Conference on Energy Internet and Energy System Integration: Connecting the Grids Towards a Low-Carbon High-Efficiency Energy System, El2 2020 (pp. 3068-3073). [9347211] (2020 IEEE 4th Conference on Energy Internet and Energy System Integration: Connecting the Grids Towards a Low-Carbon High-Efficiency Energy System, El2 2020).

https://doi.org/10.1109/EI250167.2020.9347211

\section{Published in:}

2020 IEEE 4th Conference on Energy Internet and Energy System Integration: Connecting the Grids Towards a Low-Carbon High-Efficiency Energy System, EI2 2020

\section{Citing this paper}

Please note that where the full-text provided on Manchester Research Explorer is the Author Accepted Manuscript or Proof version this may differ from the final Published version. If citing, it is advised that you check and use the publisher's definitive version.

\section{General rights}

Copyright and moral rights for the publications made accessible in the Research Explorer are retained by the authors and/or other copyright owners and it is a condition of accessing publications that users recognise and abide by the legal requirements associated with these rights.

\section{Takedown policy}

If you believe that this document breaches copyright please refer to the University of Manchester's Takedown Procedures [http://man.ac.uk/04Y6Bo] or contact uml.scholarlycommunications@manchester.ac.uk providing relevant details, so we can investigate your claim.

\section{OPEN ACCESS}




\section{Automatic detection and mapping of solar photovoltaic arrays with deep convolutional neural networks in high resolution satellite images}

\author{
$1^{\text {st }}$ Kaiji He \\ Department of Electrical and Electronic Engineering \\ University of Manchester \\ Manchester, UK \\ kaiji.he@postgrad.manchester.ac.uk
}

\author{
$2^{\text {nd }}$ Long Zhang \\ Department of Electrical and Electronic Engineering \\ University of Manchester \\ Manchester, UK \\ long.zhang@manchester.ac.uk
}

\begin{abstract}
The locations and capacities of household rooftop solar photovoltaic (PV) arrays are important for power grid planning. However, it is hard to collect such information manually as a significant number of PV arrays are distributed dispersedly in residential areas. With the development of deep learning model on image recognition, it brings an opportunity to build an intelligent detector that is able to automatically identify and delineate PV arrays in satellite images. Convolutional neural networks (CNN) are ideally suited for this task as CNN has capability of capturing spatial information of digital images by convolution operation. In this work, we trained a deep CNN with manually annotated satellite images taken from a region of Manchester (UK), and validated our detector with another set of satellite images taken from the same city. Our results indicate that the detector is capable to identify PV arrays with a high accuracy and delineate them in pixel-wise with high precision, showing the feasibility of our approach.
\end{abstract}

Index Terms-deep convolutional neural networks, mask RCNN, photovoltaic arrays, satellite images

\section{INTRODUCTION}

In recent decades, solar power has become one of the most important power sources all over the world due to the increasing popularity of renewable energy. By the end of February 2020, there are 13,421MW solar photovoltaic $(\mathrm{PV})$ arrays installed in UK, and 55.1 percent of which are small-scale PV array systems (typical $4 \mathrm{~kW}$ ) distributed dispersedly in residential areas ${ }^{1}$. The detailed information about distributed PV arrays, including their location, size, shape, and capacity, is appealing to energy suppliers as well as government agencies since it is essential in power system integration, operation, planning and policy making. However, such detailed information is not always up-to-date due to the fact that most of the databases are collected from third parties or surveys. In addition, it is time-consuming and expensive to collect the information for a great number of household PV arrays.

In order to increase the efficiency of gathering information for PV arrays, some attempts have been made to achieve PV

${ }^{1}$ https://www.gov.uk/government/statistics/solar-photovoltaics-deployment

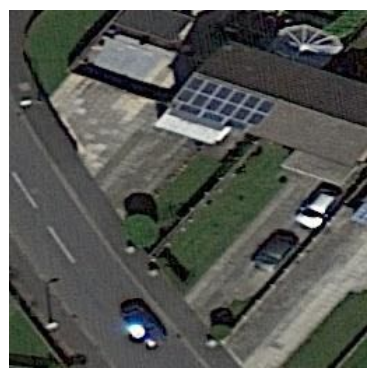

(a)

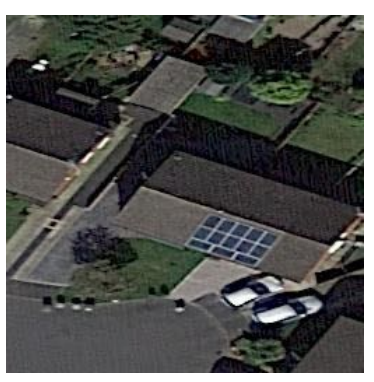

(b)
Fig. 1. Small-scale PV arrays installed in residential areas.

array identification and mapping in high resolution aerial imagery via image recognition techniques. [1] and [2] investigate the feasibility of using Random Forest (RF) classifier to build PV array detector based on satellite images and their detector is the first of their kind. They also indicate that the detection accuracy can be improved by using pre-processed image features such as local colour statistic (LCS), texton features and etc. In [3], a shallow convolutional neural network (CNN) architecture is attached to RF to form a cascade detection architecture, where $\mathrm{CNN}$ is used to refine the detection results from RF. This method greatly improves the PV array detection accuracy. But the limited depth of CNN makes it difficult for this detector to fully leverage the capability of CNN. [4] applies a deep CNN to detect PV array, yielding a higher precision than [3] based on the same dataset. The CNNbased detectors mentioned above are effective at identifying PV arrays in aerial images, but less effective at delineating them precisely. Because their CNN structures leverage downsampling operation and fully connected layers which cannot remain the spatial information of objects in digital images. To overcome this problem, [5] integrates feature maps from different stages of CNN to produce a prediction map. This approach achieves PV array mapping by pixel-wise prediction based on hierarchically integrated feature maps. In addition, [6] adopts SegNet [7], a neural network architecture specially 
designed for semantic segmentation, to build a detector for identifying PV arrays. The detectors in [5] and [6] are built via some typical semantic segmentation methods which are capable to predict precise shape and size of a single PV array. They process input image with convolution operation and then calculate the probability of belonging to a PV array for each pixel. Indeed, pixel-wise classification is necessary for resolving the shape and size of PV arrays as its output indicates the class of each pixel. However, its output does not specify which group of pixel corresponds to a real-world PV array. Although it is feasible to apply external statistic methods to group neighbouring pixels with high probabilities into PV array detections [6], this may lead to significant computation burden when dealing with a great number of images. Besides, those existing CNN-based PV array detectors achieve PV arrays mapping inefficiently as they implement segmentation on a whole input image rather than pixel regions where PV arrays present.

In this paper, we propose an approach to build a detector that can identify and delineate the PV arrays in satellite imagery simultaneously. This detector is built on top of Mask R-CNN [8] which is specialized in instance segmentation tasks. A typical feature of Mask R-CNN is that it first localises objects via region proposals and then classify and segment the objects at the same time. Its backbone has a hierarchically integrated structure so that object features are comparably strong in both high level and low level. Its capability of object detection and segmentation in satellite images has been actively studied [9] [10]. To the best of our knowledge, this is the first time to implement Mask R-CNN on PV array detection. We also compare our detector with SegNet-based detector by evaluating their pixel-wise and object-wise detection performance. These two detectors were trained with a large set of PV array images annotated manually and they were evaluated on images taken from complex urban scenes. The experiments show that our detector is more feasible to achieve PV array identification as it can output localisation and mapping of individual PV array simultaneously, which is more informative than PV array mapping by pure image segmentation.

This paper is organized as follows. Section II introduces the training and validation images for our detector. Section III describes rationales and model training of Mask R-CNN and SegNet. Section IV describes the experiment design and results. Section $\mathrm{V}$ presents the conclusions.

\section{TRAINING AND VALIDATION IMAGE DATASET}

The satellite images used in the experiments are orthorectified images with RGB channels collected from Google Earth. We downloaded a large number of satellite images encompassing a region of Manchester with $89 \mathrm{~km}^{2}$ surface area. Each image has a size of $256 \times 256$ and the image resolution is 0.3 meter. Images containing PV arrays are picked out manually, and the PV arrays are annotated by polygons. Those images are randomly split into two groups, a training set and a validation set. In total, there are 6990 PV array images.
5873 of them are used for training and the rest of them are used for validation.

TABLE I

TRAINING AND VALIDATION DATASET.

\begin{tabular}{c|c|c}
\hline Image dataset & Number of images & Number of PV array annotations \\
\hline Training set & 5873 & 10185 \\
\hline Validation set & 1117 & 1773 \\
\hline
\end{tabular}

\section{IMPLEMENTATION OF MASK R-CNN AND SEGNET}

\section{A. Mask R-CNN architecture}

Mask R-CNN is a two-stage model that performs object detection and segmentation on a set of regions extracted from input images. The first stage is Region Proposal Network (RPN) [11], while the second stage is designed for object detection and segmentation. Both stages use the same backbone so as to reduce the computation burden. Besides, this model has three outputs, namely object classification, bounding-box location and object mask presentation. The overall structure of Mask R-CNN is depicted in Fig. 2.

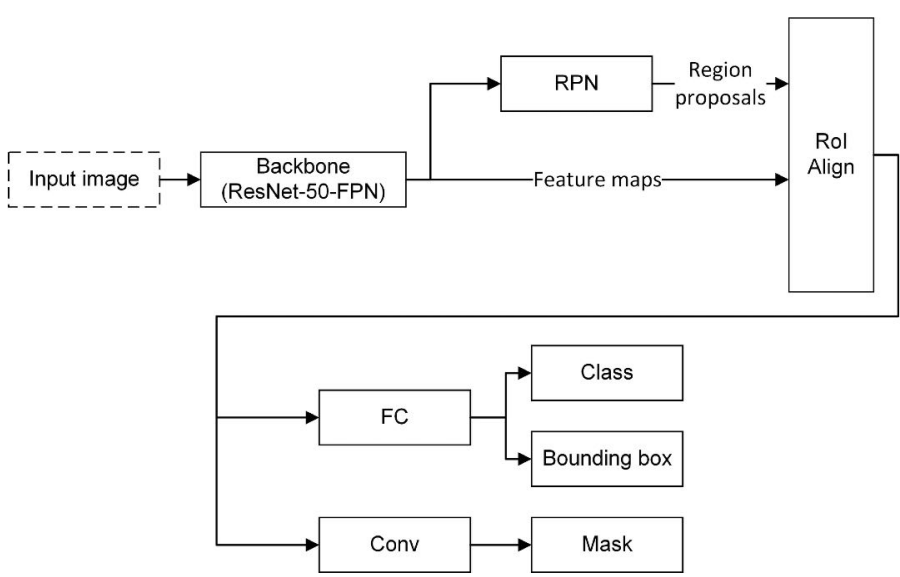

Fig. 2. Overall structure of Mask R-CNN [8]. The bottom boxes are network head where FC stands for fully connected layers and Conv stands for convolutional layers.

1) Backbone: The main role of backbone is to extract feature maps from input images. In this paper, ResNet-50Feature Pyramid Networks (FPN) is adopted to serve as the backbone. This deep CNN architecture ensures the detector's capability of identifying PV arrays with various scale. ResNet [12] is used to prevent the degradation of training accuracy when the depth of convolutional layers is increased to an extremely large extent. The depth of layer is an important factor for $\mathrm{CNN}$, as increasing layer depth introduces more weights to the deep learning model, allowing the model to fit complex input data as much as possible. However, increasing network depth makes training accuracy saturated and then degraded due to the gradient vanishing problem during back propagation. ResNet solves this problem by using residual network that comprises two fundamental components, the 
building block and bottleneck block. Both of them are utilized to model the aggregation of residual mapping and identity mapping.

FPN [13] is used to construct feature pyramids by inherent pyramidal hierarchy of CNN. Feature pyramids are widely used in object detection tasks because the multi-scale feature representation guarantees that features are comparably strong in all pyramid levels. FPN combines the low-level features and high-level features from the plain network via a bottomup pathway, a top-down pathway and lateral connections. The bottom-up pathway performs standard convolution operation done by successive convolutional layers, while the top-down pathway upsamples the spatial resolution of feature maps from high level pyramids. The upsampled feature maps are laterally connected to the feature maps with the same spatial size produced by bottom-up pathway. Given the feature pyramids produced by FPN, the prediction results for object detection tasks are made in each pyramid level.

2) Region proposal networks (RPN): RPN is particularly designed for class-agnostic region proposal extraction from input images. After the backbone extracts feature maps from input images and feeds them into RPN, RPN predicts multiple region proposals simultaneously in a sliding window fashion based on some reference boxes called anchors. Anchors are fixed size bounding-boxes located at the center of each sliding window location. Usually, they have various scales and aspect ratios, which ensures that objects with different size and shape are all considered during region proposal prediction. Positive or negative labels are assigned to anchors based on their Intersection of Unions (IoU) with ground-truth boxes. The output of RPN consists of box-classification and boxregression. The box-classification refers to the probability of a predicted region proposal being an object, and the boxregression refers to the predicted coordinate offsets of region proposals. RPN outputs a great amount of region proposals, but only a certain amount of high scoring region proposals denoted as Region of Interests (RoIs) are fed into the second stage.

3) Object classification, localisation and mask prediction: The second stage of Mask R-CNN starts with RoI-align layer which is designed to process feature maps of RoIs. At first, RoIs are projected onto feature maps produced by backbone. Then, every projection is transformed into a grid where bins have the same spatial size, and the RoI-align layer leverages bilinear interpolation to calculate the value of several regularly sampled points inside each bin. After that, RoI-align layer performs max pooling or average pooling over all the samples points in each bin. The resultant tensors are propagated to three parallel layers, which are object classification layer, boundingbox regression layer, and mask prediction layer. The object classification layer can categorize the objects inside RoIs, while bounding-box layer can localise those objects. Both of them are achieved by a set of fully connected layers. However, mask prediction layer comprises successive convolutional layers and a deconvolutional layer since convolutional layer can ensure high spatial resolution which is essential to precise mask presentation. Based on the final outputs of Mask R-CNN, the loss function is defined as a summation of classification loss, bounding box-regression loss and mask prediction loss.

\section{B. Training of Mask R-CNN}

Training of Mask R-CNN is run on python using a single NVIDIA Tesla 16GB GPU. The weights are updated by stochastic gradient descent with 1 image as a mini-batch. After trying different sets of parameters, the learning rate, momentum and weight decay are set as 0.0001, 0.9 and 0.0001 respectively, which produces the best detection performance. Considering the PV arrays have various shape and size, the anchor scales and ratios are set as $\{8,16,32,64,128\}$ and $\{0.5,1,2\}$.

Aiming to improve the training efficiency, the weights are initialised by pre-trained $\mathrm{COCO}$ weights ${ }^{2}$. The pre-trained weights allow the loss converge more quickly. There are two training approaches considered in the experiments. The first approach only trains the network head of Mask R-CNN, leaving the rest of weights unchanged. The second approach trains all the layers including the backbone and the network head. Both of them stop after 400 epochs.

\section{SegNet architecture}

SegNet has a single stage CNN architecture and it is specialized at semantic segmentation. Its structure can be divided into two parts. The first part is called "encoder" which is a typical CNN architecture. It consists of successive convolutional layers and performs down-sampling by several max-pooling layers. The second part is called "decoder" and it is designed to produce segmentation mask that has the same size as the input image. The decoder comprises a set of convolutional layers and several upsampling layers. Each layer in decoder is paired with a corresponding layer in encoder. The upsampling layers up-sample the low-resolution feature maps according to the max-pooling index from their counterparts in encoder, yielding sparse feature maps with extended spatial size. The decoder ends with a soft-max classifier which is applied to each feature vector from the final feature map. In the experiment, there are two classes, namely, PV array and background. As a result, the final output of decoder is a $W \times H \times 2$ tensor where $W \times H$ is the spatial size of input image.

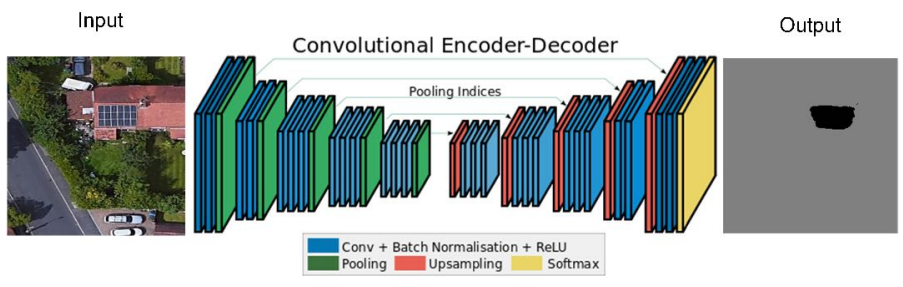

Fig. 3. Overall structure of SegNet [7].

\footnotetext{
${ }^{2}$ https://github.com/matterport/Mask_RCNN
} 


\section{Training of SegNet}

Similar to the training of Mask R-CNN, the training of SegNet is also implemented with python and run on a single NVIDIA Tesla 16GB GPU. Since the CNN structure of SegNet is relatively simpler than that of Mask R-CNN, there is no need to use pre-trained weights to train SegNet. Therefore, the weights of SegNet are initialized by gaussian distribution and updated by stochastic gradient descent. The batch size is set as 2 , and the learning rate, momentum and weight decay are set as $0.001,0.9,0.0001$ respectively. The training stops at 50 epochs.

\section{EXPERIMENT DESIGN AND RESULTS}

\section{A. Evaluation metric}

We use precision-recall (PR) curve for evaluating the detector performance on PV array detection. The PR curve is a common metric for evaluating segmentation tasks and it is based on the IoU between the ground-truth annotations and detections. Given a ground-truth mask $\alpha$ and predicted pixel region $\beta$, the IoU is given by:

$$
I o U=\frac{\alpha \cap \beta}{\alpha \cup \beta}
$$

If IoU is larger than a pre-defined threshold, the corresponding predicted mask is regarded as true positive, otherwise false positive. In the experiments, the threshold is set as 0.5. If the number of true positive and false negative are known, then the precision and recall can be given by:

$$
\begin{aligned}
& \text { Precision }=\frac{\mathrm{TP}}{\mathrm{TP}+\mathrm{FP}} \\
& \text { Recall }=\frac{\mathrm{TP}}{\mathrm{TP}+\mathrm{FN}}
\end{aligned}
$$

where TP, FP, FN are abbreviations for true positive, false positive and false negative.

The precision describes the detector's ability of identifying PV arrays accurately and it is determined by the percentage of accurate positive predictions. The recall indicates the detector's ability of finding all the PV arrays and it is determined by the percentage of true positive cases among all ground-truth cases.

\section{B. Experiment results}

For Mask R-CNN detector, two training approaches mentioned in Section III-B are applied to the detector respectively. Training network head of Mask R-CNN costs 12 hours, while training all the layers costs 16 hours. Their training losses are depicted in Fig. 4(a). Since the networks are initialized with pre-trained weights, the training losses decrease rapidly at the beginning. It is worth mentioning that training all layers yields smaller training loss than training the network head. We conjecture that although the pre-trained $\mathrm{COCO}$ weights accelerate the training process, it is not suited for the image pattern of PV array images. Training of SegNet detector takes about 4 hours. Its training loss is shown in Fig. 4(b).

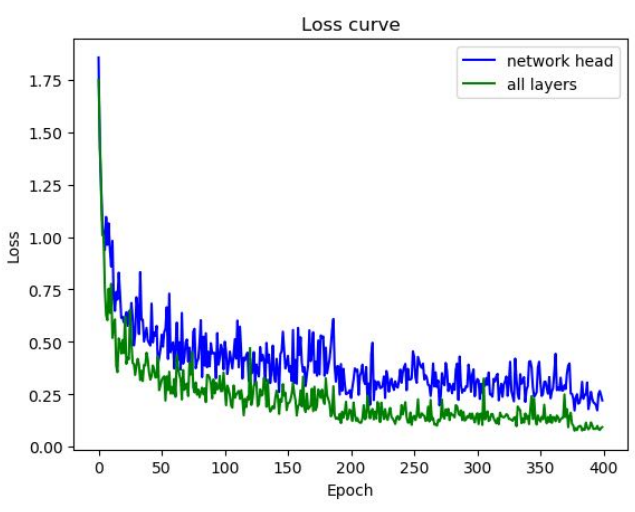

(a)

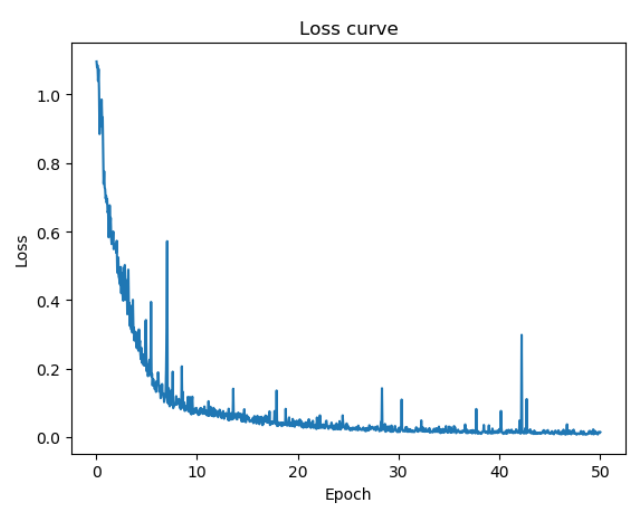

(b)

Fig. 4. (a) Training loss of Mask R-CNN model, (b) Training loss of SegNet model.

Two trained detectors are applied to the same validation images separately. Due to more complicated CNN structure, Mask R-CNN detector spends more time to process one image than SegNet detector.

TABLE II

IMAGE PROCESSING TIME.

\begin{tabular}{c|c|c}
\hline Models & Mask R-CNN & SegNet \\
\hline Average processing time & $1.151 \mathrm{~s}$ & $\mathbf{0 . 6 5 8 s}$ \\
\hline
\end{tabular}

Given the validation set, the Mask R-CNN detector can successfully identify and delineate most of the PV arrays accurately as shown in Fig. 7. The PR curves are shown in Fig. 5. The detector with layers all trained have better performance on validation set than that with trained network head. The former one has average precision (AP) up to $95.66 \%$, while the latter one only has $93.02 \%$ AP. Both of them can achieve a recall rate of around $95 \%$. However, there are still a certain amount of false alarms as the PR curves in Fig. 5 decrease sharply when recall approaches 1 . This is because some true positive samples have low probability. In addition, some objects (e.g. glass window) are similar to PV arrays in terms of size, shape and colour as shown in Fig. 6(a), which makes it 
difficult for the detector to classify. Moreover, the recall cannot reach 1 due to the fact that a small number of PV arrays are not detected as depicted in Fig. 6(b)(c)(d).

Compared to the detection performance of Mask R-CNN detector, the SegNet detector has lower average precision $(80.31 \%)$ as shown in Fig. 5. It only finds $80 \%$ of all the ground-truth samples. Meanwhile, the detections of SegNet detector cannot match the ground-truth PV arrays precisely (see Fig. 7), which could heavily degrade the estimation of the shape and size of PV arrays. Similar to Mask R-CNN detector, SegNet detector also fails to distinguish the objects that have appearance similar to PV arrays.

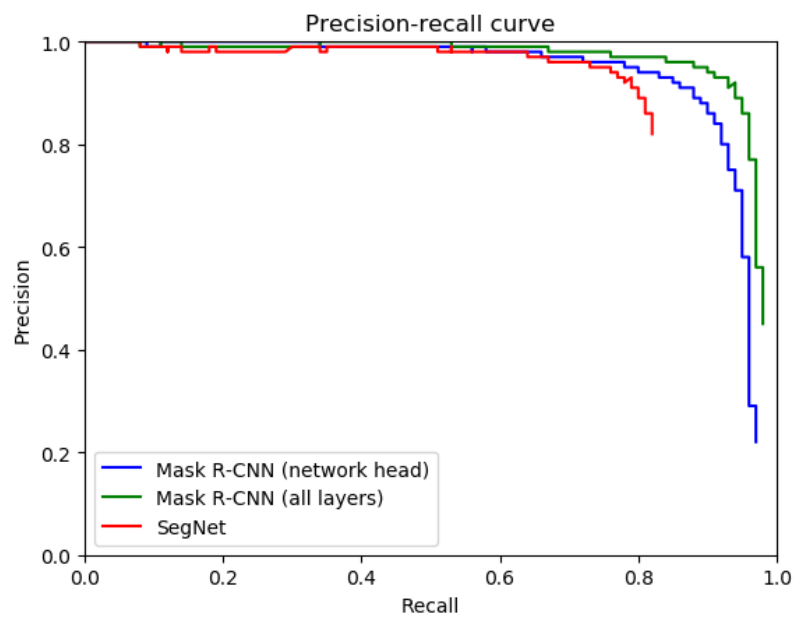

Fig. 5. PR curves of validation results.

\section{CONCLusions}

In this work, we investigate a deep $\mathrm{CNN}$ architecture and its implementation on PV arrays identification and mapping in satellite images. We use Mask R-CNN to build a detector that can accurately classify and delineate PV arrays and compare our detector with another detector from [6]. The training images and validation images are collected from a region of Manchester, UK. The networks are initialised with pre-trained weights, and there are two training approaches considered including training all the layers and training the network heads. Our detector can achieve an average precision of $95.66 \%$, showing the feasibility of using deep CNN to build a PV array detector.

\section{REFERENCES}

[1] J. M. Malof, R. Hou, L. M. Collins, K. Bradbury, and R. Newell, "Automatic solar photovoltaic panel detection in satellite imagery," in 2015 International Conference on Renewable Energy Research and Applications (ICRERA). IEEE, 2015, pp. 1428-1431.

[2] J. M. Malof, K. Bradbury, L. M. Collins, R. G. Newell, A. Serrano, $\mathrm{H}$. Wu, and S. Keene, "Image features for pixel-wise detection of solar photovoltaic arrays in aerial imagery using a random forest classifier," in 2016 IEEE International Conference on Renewable Energy Research and Applications (ICRERA). IEEE, 2016, pp. 799-803.

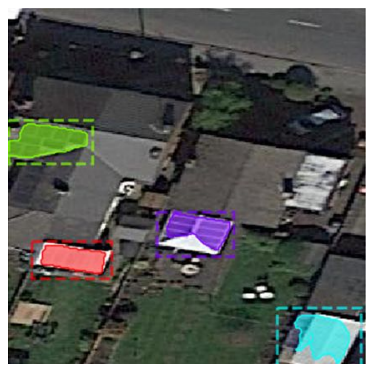

(a)

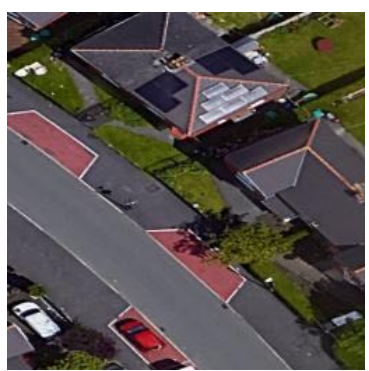

(c)

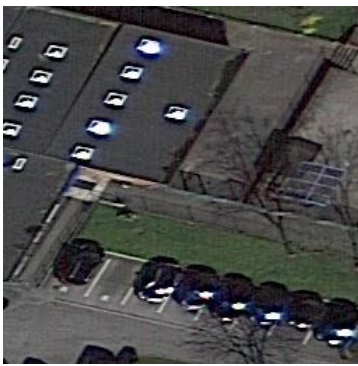

(b)

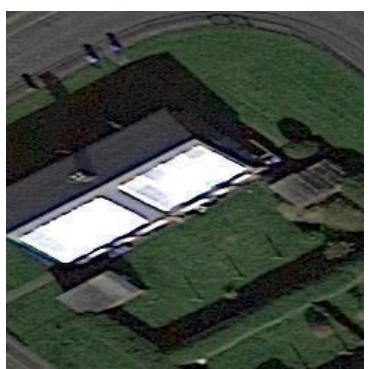

(d)
Fig. 6. (a) Glass window covered in purple mask is falsely identified as PV array, (b) Detector fails to detect PV array shadowed by tree, (c) Detector fails to detect PV array with irregular shape, (d) Detector fails to detect PV covered by reflection.

[3] J. M. Malof, L. M. Collins, K. Bradbury, and R. G. Newell, "A deep convolutional neural network and a random forest classifier for solar photovoltaic array detection in aerial imagery," in 2016 IEEE International Conference on Renewable Energy Research and Applications (ICRERA). IEEE, 2016, pp. 650-654.

[4] J. M. Malof, L. M. Collins, and K. Bradbury, "A deep convolutional neural network, with pre-training, for solar photovoltaic array detection in aerial imagery," in 2017 IEEE International Geoscience and Remote Sensing Symposium (IGARSS). IEEE, 2017, pp. 874-877.

[5] J. Yuan, H.-H. L. Yang, O. A. Omitaomu, and B. L. Bhaduri, "Largescale solar panel mapping from aerial images using deep convolutional networks," in 2016 IEEE International Conference on Big Data (Big Data). IEEE, 2016, pp. 2703-2708.

[6] J. Camilo, R. Wang, L. M. Collins, K. Bradbury, and J. M. Malof, "Application of a semantic segmentation convolutional neural network for accurate automatic detection and mapping of solar photovoltaic arrays in aerial imagery," arXiv preprint arXiv:1801.04018, 2018.

[7] V. Badrinarayanan, A. Kendall, and R. Cipolla, "Segnet: A deep convolutional encoder-decoder architecture for image segmentation," IEEE transactions on pattern analysis and machine intelligence, vol. 39, no. 12, pp. 2481-2495, 2017.

[8] K. He, G. Gkioxari, P. Dollár, and R. Girshick, "Mask r-cnn," in Proceedings of the IEEE international conference on computer vision, 2017, pp. 2961-2969.

[9] K. Zhao, J. Kang, J. Jung, and G. Sohn, "Building extraction from satellite images using mask r-cnn with building boundary regularization." in CVPR Workshops, 2018, pp. 247-251.

[10] X. Wang, T. Xie, and L. Chen, "Urban village identification from city-wide satellite images leveraging mask r-cnn," in UK Workshop on Computational Intelligence. Springer, 2019, pp. 166-172.

[11] S. Ren, K. He, R. Girshick, and J. Sun, "Faster r-cnn: Towards real-time object detection with region proposal networks," in Advances in neural information processing systems, 2015, pp. 91-99.

[12] K. He, X. Zhang, S. Ren, and J. Sun, "Deep residual learning for image recognition," in Proceedings of the IEEE conference on computer vision and pattern recognition, 2016, pp. 770-778.

[13] T.-Y. Lin, P. Dollár, R. Girshick, K. He, B. Hariharan, and S. Belongie, "Feature pyramid networks for object detection," in Proceedings of the IEEE conference on computer vision and pattern recognition, 2017, pp. $2117-2125$ 


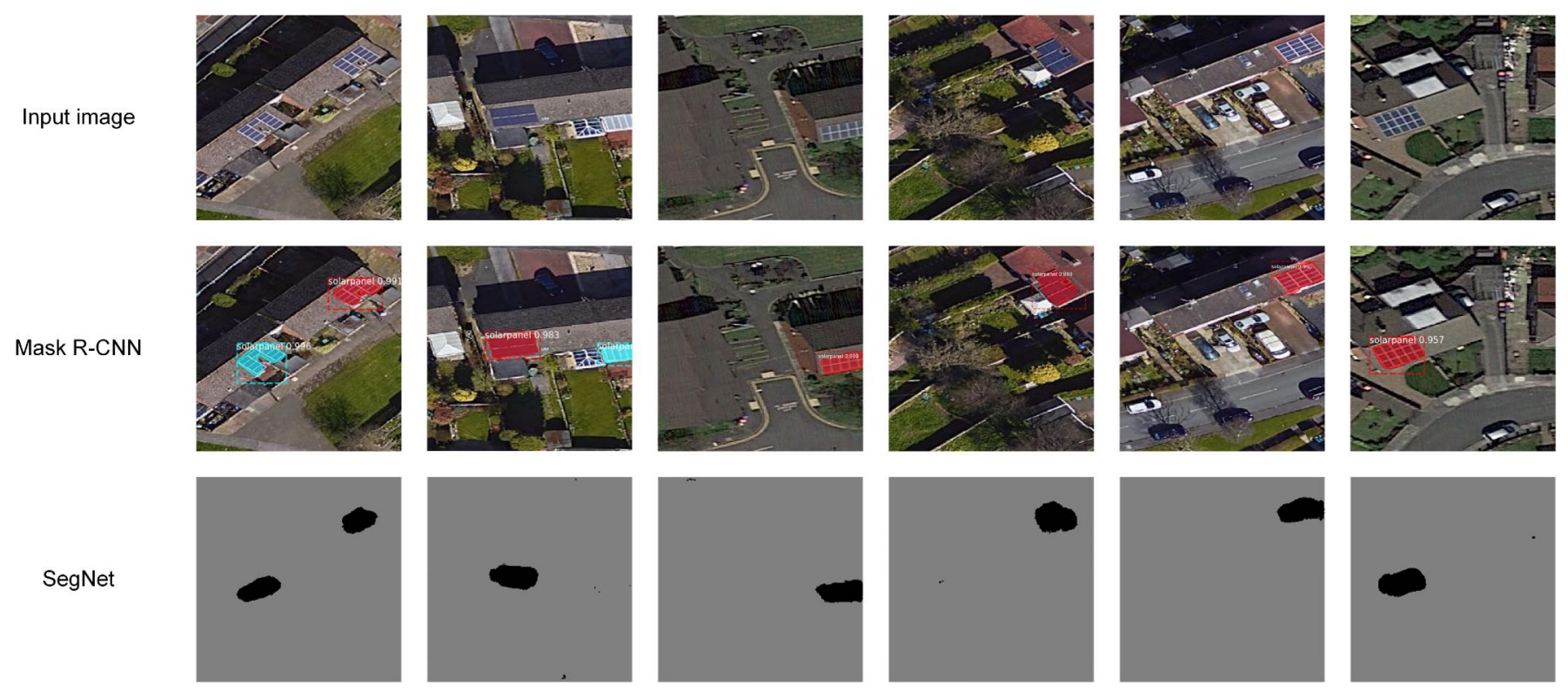

Fig. 7. Examples of validation of Mask R-CNN-based detector and SegNet-based detector.
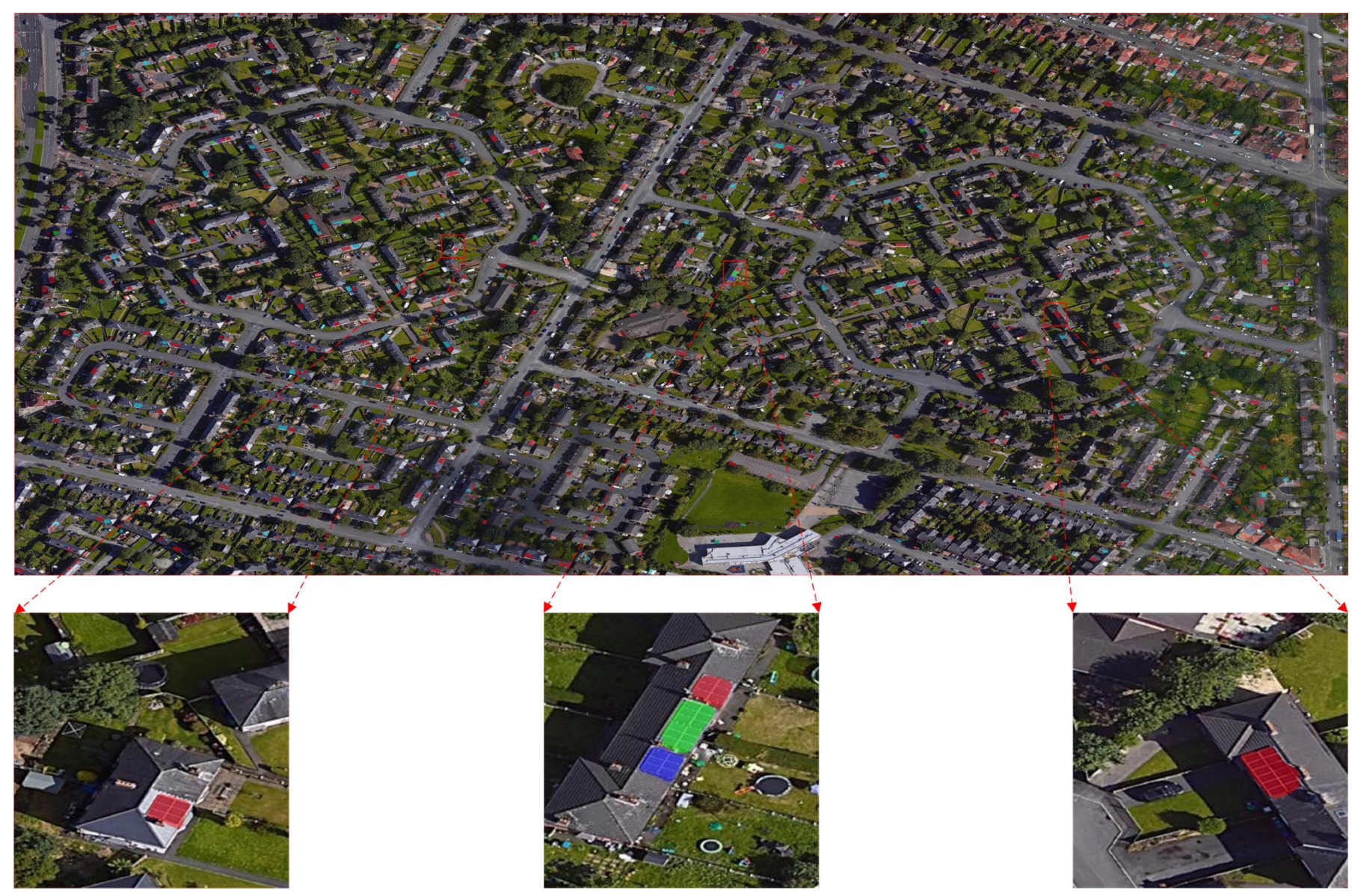

Fig. 8. Validation examples of Mask R-CNN-based detector where image tiles produced by detector are combined together. 